\title{
GMR
}

\section{Apoptosis, proliferation, and morphology during vein graft remodeling in rabbits}

\author{
L. Wan, S.H. Dai, S.Q. Lai, L.Q. Liu, Q. Wang, H. Xu, W.J. Wang and \\ J.C. Liu \\ Department of Cardiovascular Surgery, \\ First Affiliated Hospital of Nanchang University, Nanchang, China \\ Corresponding author: J.C. Liu \\ E-mail: liujichun999@163.com
}

Genet. Mol. Res. 15 (4): gmr.15048701

Received April 7, 2016

Accepted August 1, 2016

Published October 5, 2016

DOI http://dx.doi.org/10.4238/gmr.15048701

Copyright (C) 2016 The Authors. This is an open-access article distributed under the terms of the Creative Commons Attribution ShareAlike (CC BY-SA) 4.0 License.

\begin{abstract}
Neo-intima development and atherosclerosis limit the long-term use of vein grafts for revascularization of ischemic tissues. Recently, studies have confirmed that proliferating cell nuclear antigen (PCNA) plays an important role in cell proliferation. Our research confirmed that 28 days after vein transplantation, PCNA expression increases significantly. Using rabbits, rather than rodents, for a more representative model of human vein grafts, we aimed to establish a time course of changes in cell proliferation and apoptosis using morphometric and immunohistochemical analyses, western blot, terminal deoxynucleotidyl transferase dUTP nick end labeling, and transmission electron microscopy (TEM). The external jugular veins of 42 healthy purebred male New Zealand white rabbits were grafted onto their common carotid arteries. The rabbits were divided into seven groups, with vein grafts being harvested before surgery, and at 1, 3, 7, 14, 28, and 90 days afterwards. The extent of stenosis and apoptosis, PCNA protein levels, and TEM morphology were
\end{abstract}


subsequently examined. Intimal thickness was slightly decreased 1 day following surgery, but then increased continuously until the 90th day. Western blot and immunohistochemistry both indicated lowered PCNA expression on day 1, although levels subsequently increased, peaking at 7 days post-surgery. After surgery, apoptosis was lowest on day 7 , and remained low thereafter. TEM revealed signs of apoptosis as vein graft restenosis progressed. Proliferation and apoptosis cooccurred following grafting, indicating that both processes were involved in vein graft remodeling. Apoptosis levels were highest between days 1 and 3 after surgery, whereas proliferation culminated on the 7 th day.

Key words: Proliferating cell nuclear antigen; Vein graft; Restenosis

\section{INTRODUCTION}

The great saphenous vein remains the most commonly used vessel in coronary artery bypass grafting. However, the patency rate of such grafts is much lower than that of the artery itself, and no effective therapy for the prevention of long-term vein graft restenosis exists (Jeremy et al., 2011). Increasing evidence indicates that regulation of the cell cycle and apoptosis involves a complex set of gene networks. During vein graft remodeling, several abnormal changes in these networks have been noted (Wan et al., 2006a,b; Jiang et al., 2007). Apoptosis has been shown to occur in in vitro cultures of vascular smooth muscle cells (VSMCs), as well as in in vivo arterial systems, and plays an important role in vascular remodeling. However, studies concerning apoptosis in this process have been largely limited to arterial systems, with few having examined vein grafts.

In this investigation, we assessed the degree of stenosis, transmission electron microscopy (TEM) morphology, and proliferating cell nuclear antigen (PCNA) protein and apoptosis levels at different time points following vein graft surgery. Moreover, we characterized a time course of both proliferation and apoptosis because of vein grafting. The purpose of this study was to systematically analyze modulation and intervention time points for the prevention of vein graft restenosis.

\section{MATERIAL AND METHODS}

\section{Experimental animals and groups}

Forty-two healthy purebred male New Zealand White rabbits weighing 2.5-3.0 kg and aged 4-5 months were randomly divided into seven groups (T1, T2, T3, T4, T5, T6, and T7), each containing six rabbits.

Animal breeding services and the animal model itself were provided by the Department of Laboratory Animal Science of Nanchang University, whose conditions meet the national experimental standard. The laboratory temperature was maintained at $22 \pm 1^{\circ} \mathrm{C}$. Euthanasia was performed by intravenous injection of $2 \mathrm{mg} / \mathrm{kg}$ potassium chloride solution under anesthesia, conforming to the guidance on the treatment of experimental animals issued by the Ministry of Science and Technology of the People's Republic of China.

Genetics and Molecular Research 15 (4): gmr.15048701 


\section{Principal reagents}

A terminal deoxynucleotidyl transferase dUTP nick end labeling (TUNEL) cell apoptosis detection kit was purchased from Promega (Madison, WI, USA). PCNA mouse monoclonalanti-rabbit primary antibodies were obtained from SAB (College Park, MD, USA). Horseradish peroxidase-labeled goat anti-mouse immunoglobulin $\mathrm{G}$ antibody and Super Signal West Pico chemiluminescent substrate were manufactured by Beijing Zhong Shan-Golden Bridge Biological Technology Co. (Beijing, China) and Pierce (Rockford, IL, USA), respectively.

\section{Methods}

\section{Preoperative preparation and anesthesia}

Routine preoperative preparation was as follows: experimental animals were fasted for $4 \mathrm{~h}$ and prevented from drinking for $6 \mathrm{~h}$ prior to surgery. They were then administered an ip injection of $200 \mathrm{IU} / \mathrm{kg}$ heparin, and an im injection of $30 \mathrm{mg} / \mathrm{kg}$ ketamine to induce anesthesia. Anesthesia was maintained by injecting the auricular vein with $3 \%$ pentobarbital (30 mg/kg).

All animal procedures followed institutional guidelines for the care and use of laboratory animals. This study protocol was approved by the local Ethics Committee (Institutional Review Board of the First Affiliated Hospital of Nanchang University).

\section{Establishing the autologous vein graft restenosis model}

We established a rabbit model of restenosis using the method described by Wan et al. (2006a). After $i v$ injection of heparin, the right common carotid artery and jugular vein were carefully exposed through a vertical midline neck incision. Under an operating microscope, a 4-cm segment of the jugular vein was resected from the surrounding tissue using a no-touch technique, removed from the animal, and rinsed with phosphate-buffered saline. The common carotid artery was then dissected, and its flow interrupted with microvascular clips. End-to-end anastomosis of the reversed vein graft and carotid artery was performed with approximately 10 interrupted sutures per anastomosis using 8-0 nylon monofilament (Ethicon, Somerville, NJ, USA).

\section{Collecting and handling vein graft specimens}

For groups T1, T2, T3, T4, T5, T6, and T7, the vein grafts were harvested before surgery, and at $1,3,7,14,28$, and 90 days afterwards, respectively. Each vein graft was divided into three pieces as follows: one was stored at $-80^{\circ} \mathrm{C}$ until needed for western blot analysis; one was fixed in $4 \%$ paraformaldehyde for hematoxylin and eosin (H\&E) staining, immunohistochemistry, and the TUNEL apoptosis assay; and one was fixed in glutaraldehyde for TEM.

\section{Detection methods}

\section{Morphological observation}

Following H\&E staining, the sections were examined by light microscopy. The

Genetics and Molecular Research 15 (4): gmr.15048701 
thicknesses of the tunica intima and tunica media plus the tunica adventitia were measured using Motic digital medical image analysis system (Motic Ltd., China). A cross-section of the vessel being measured at six evenly spaced points (points " $2,4,6,8,10$, and 12" correspond to a 12-part division, as on a clock face). Thus, six measurements were recorded in total, the average of which was calculated. The intima thickness was calculated as that between the internal elastic lamina and the vascular lumen. The combined thickness of the media and adventitia was calculated as that between the internal elastic membrane and the outer vessel wall.

Segments were stained with H\&E and examined by light microscopy. Thicknesses were quantified using an Olympus CX-21 microscope with a color digital camera (Moticam 2006) and Motic digital medical image analysis system (Motic Ltd., China) by two different observers blinded to the time point at which each sample was taken.

\section{Western blot analysis}

As PCNA localizes to the nucleus, nucleoprotein was extracted and analyzed by western blotting, using lamin B as an internal reference. Electrophoresis was initially conducted under $80 \mathrm{~V}$, until the bromophenol blue solution reached the junction of the stacking and resolving gels (after approximately $20 \mathrm{~min}$ ). The voltage was then increased to $100 \mathrm{~V}$, and electrophoresis was stopped when the bromophenol blue solution arrived at the end of the gel (after approximately $1 \mathrm{~h} 20 \mathrm{~min}$ ). Under a constant $20-\mathrm{mA}$ current, proteins were transferred to a polyvinylidene fluoride membrane in a $4^{\circ} \mathrm{C}$ refrigerator overnight. The grayscale ratio of the band of interest to the internal reference band was subsequently calculated.

\section{Immunohistochemistry}

Tissue sections were deparaffinized in CitriSolv (Fisher Scientific, Loughborough, UK) and rehydrated in serially diluted alcohol solutions. Antigen retrieval was performed in citrate buffer, $\mathrm{pH} 6.0$, for $40 \mathrm{~min}$ at $90^{\circ} \mathrm{C}$. Endogenous peroxidase activity was blocked with $3 \% \mathrm{H}_{2} \mathrm{O}_{2}$ in aqueous solution for $5 \mathrm{~min}$, and non-specific binding was prevented by blocking with normal horse serum for $30 \mathrm{~min}$. Sections were incubated overnight with primary antibodies.

PCNA localized to the nucleus; therefore, the nuclei of positive cells had a yellowbrown appearance. Using light microscopy at 400X magnification, the percentage of positively stained cells was calculated according to the total number of cells per field. For each section, 10 fields were randomly chosen, in which positive cells were counted to determine the mean percentage expressing PCNA.

\section{TUNEL apoptosis assay}

Tissue sections were deparaffinized in CitriSolv, rehydrated in serially diluted alcohol solutions, and permeabilized with $0.5 \%$ Triton X-100 in $0.1 \%$ sodium citrate. The completely dried slices were examined under aninverted phase contrast microscope. Ten fields were photographed for each section. All images were evaluated in a randomized, doubleblinded manner. If a nucleus exhibited yellow staining, the cell was considered positive. The percentage of positive cells was measured using Image-Pro Plus6.0 (Media Cybernetics, Inc., Rockville, MD, USA).

Genetics and Molecular Research 15 (4): gmr.15048701 


\section{TEM observation}

Samples were fixed for $1 \mathrm{~h}$ in $2 \%$ glutaraldehyde dissolved in $0.15 \mathrm{M}$ phosphate buffer, $\mathrm{pH} 7.2$, followed by post-fixation for $1 \mathrm{~h}$ in $1 \%$ osmium tetroxide dissolved in $0.9 \%$ sodium chloride. Fixed material was stained en bloc in $0.5 \%$ aqueous uranyl acetate overnight, before being dehydrated in a graded acetone series and embedded in Araldite resin. Ultrathin sections $(70 \mathrm{~nm})$ were obtained using a diamond knife in an LKB ultramicrotome (LKB Instruments, Inc., Rockville, MD, USA), placed on 200-mesh copper grids, and double-stained with uranyl acetate and lead citrate. The grids were studied and micrographs taken with a Philips (Amsterdam, Netherlands) TEM 400 transmission electron microscope, operating at $80 \mathrm{kV}$.

\section{Statistical methods}

Statistical analysis was performed using SPSS19.0 (IBM Corp., Armonk, NY, USA). Measurements are reported as means \pm standard deviations. Comparisons between groups were tested by one-way analysis of variance, while multiple comparisons were assessed using the Student-Newman-Keuls method. $\mathrm{P}<0.05$ denotes statistical significance.

\section{RESULTS}

\section{Morphological observation}

Intimal thickness was found to have decreased slightly 1 day after surgery, but subsequently increased continuously until the 90th day. Combined medial-adventitial thickness increased at each time point following surgery (Figure 1).
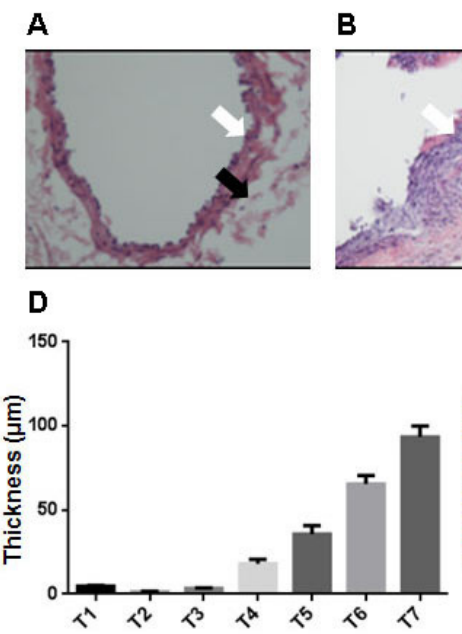

B

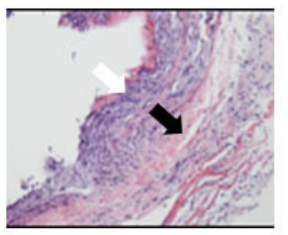

E
C
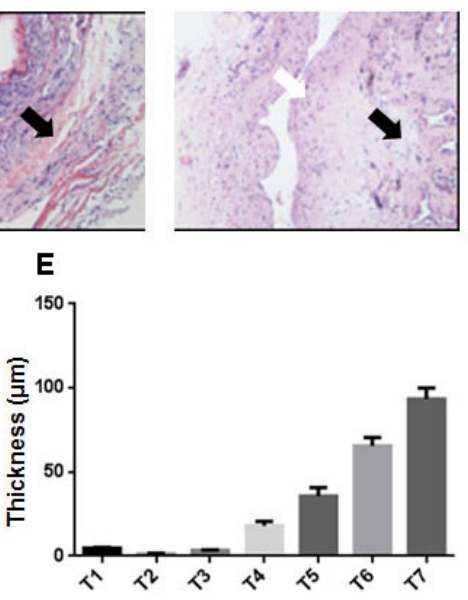

Figure 1. A.-C. Morphometric analysis of an arterialized jugular vein. D. E. Quantification of intimal and medial + adventitial thicknesses. T1, T2, T3, T4, T5, T6, and T7 represent the normal vein group, and grafts at 1, 3, 7, 14, 28, and 90 days following surgery, respectively. Each bar represents the mean \pm standard error $(\mathrm{N}=6)$. A. T1, B. T4, C. T7. The white arrow indicate tunica intima, while the black arrow indicate tunica media plus the tunica adventitia. The difference (groups T2-T7 vs T1) was statistically significant $(\mathrm{P}<0.05)$. 


\section{Western blotting analysis}

The grayscale ratio of the band of interest to the internal reference band demonstrated that PCNA expression had decreased 1 day after surgery (group T2), before increasing to a peak on the 7th day (group T4) and gradually declining thereafter (Figure 2).

A

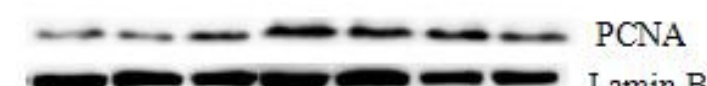

B

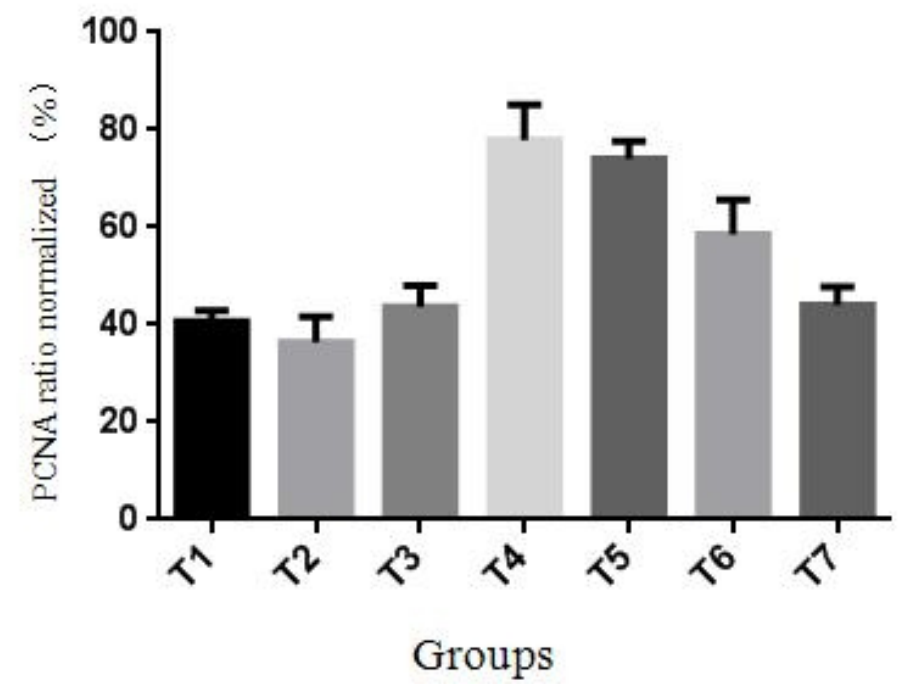

Figure 2. A. Western blotting analysis of vein tissue in each group. B. T1, T2, T3, T4, T5, T6, and T7 represent the normal vein group, and grafts at $1,3,7,14,28$, and 90 days following surgery, respectively. The difference (groups $\mathrm{T} 2-\mathrm{T} 7$ vs $\mathrm{T} 1)$ was statistically significant $(\mathrm{P}<0.05)$. PCNA = proliferating cell nuclear antigen.

\section{Immunohistochemistry}

The percentage of PCNA-positive cells in group T1 was $6.07 \pm 1.1 \%$, which decreased slightly to $5.05 \pm 0.9 \% 1$ day following surgery (group T2). By the $7^{\text {th }}$ day, this value had peaked ( $31.2 \pm 3.4 \%$; group T4), and gradually decreased after this point (Figure 3$)$. A statistically significant difference $(\mathrm{P}<0.05)$ was observed between normal (group $\mathrm{T} 1$ ) and grafted veins (groups T2-T7).

\section{TUNEL apoptosis assay}

A small number of apoptotic cells were observed in T1 vein samples $(1.49 \pm 0.2 \%)$. The rate of apoptosis was highest on the 1st day following surgery $(18.02 \pm 1.9 \%)$, and had decreased by day $3(7.29 \pm 0.7 \%)$, reaching its lowest level on the 7 th day $(2.09 \pm 0.5 \%)$ and subsequently remaining low. The percentage of apoptotic cells on days 14, 28, and 90 after surgery was $4.54 \pm 0.8,6.37 \pm 0.6$, and $6.67 \pm 1.3 \%$, respectively (Figure 4 ). 

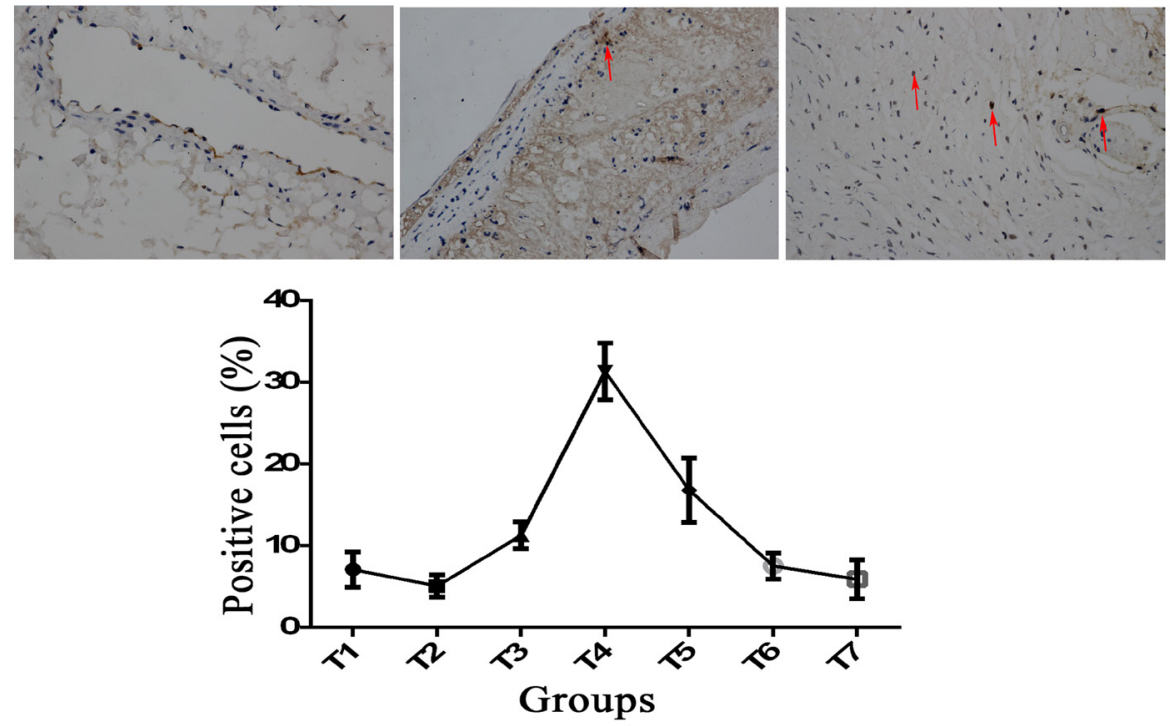

Figure 3. Immunohistochemical detection of the percentage of proliferating cell nuclear antigen-positive cells in each group. Groups T1, T2, T3, T4, T5, T6, and T7 represent normal veins, and grafts at 1, 3, 7, 14, 28, and 90 days following surgery, respectively. Arrows indicate positive cells. The difference (groups T2-T7 vs T1) was statistically significant $(\mathrm{P}<0.05)$.
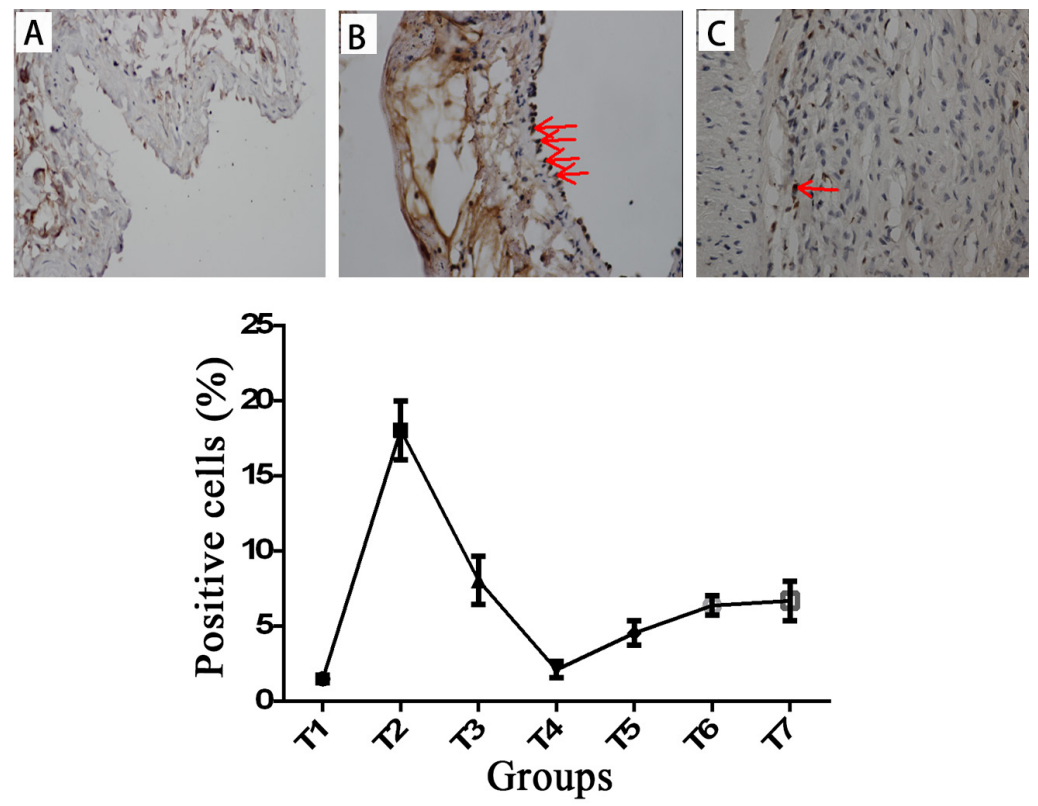

Figure 4. Terminal deoxynucleotidyl transferase dUTP nick end labeling assay of apoptotic cells in each group. T1, T2, T3, T4, T5, T6, and T7 signify normal veins, and grafts at 1, 3, 7, 14, 28, and 90 days following surgery, respectively. A. T1, B. T2, C. T4. Arrows indicate positive cells. The difference (group T2-T7 vs T1) was significant $(\mathrm{P}<0.05)$.

Genetics and Molecular Research 15 (4): gmr.15048701 


\section{TEM observation of vein graft structure}

In the vein grafts on the 1 st day (Figure 5A) and the 3rd day (Figure 5B) following surgery, apoptotic cell bodies were observed. In the vein grafts on the 7th day (Figure 5C) and the 14th day following surgery, smooth muscle cells were abundant in both the Golgi apparatus and the rough endoplasmic reticulum.
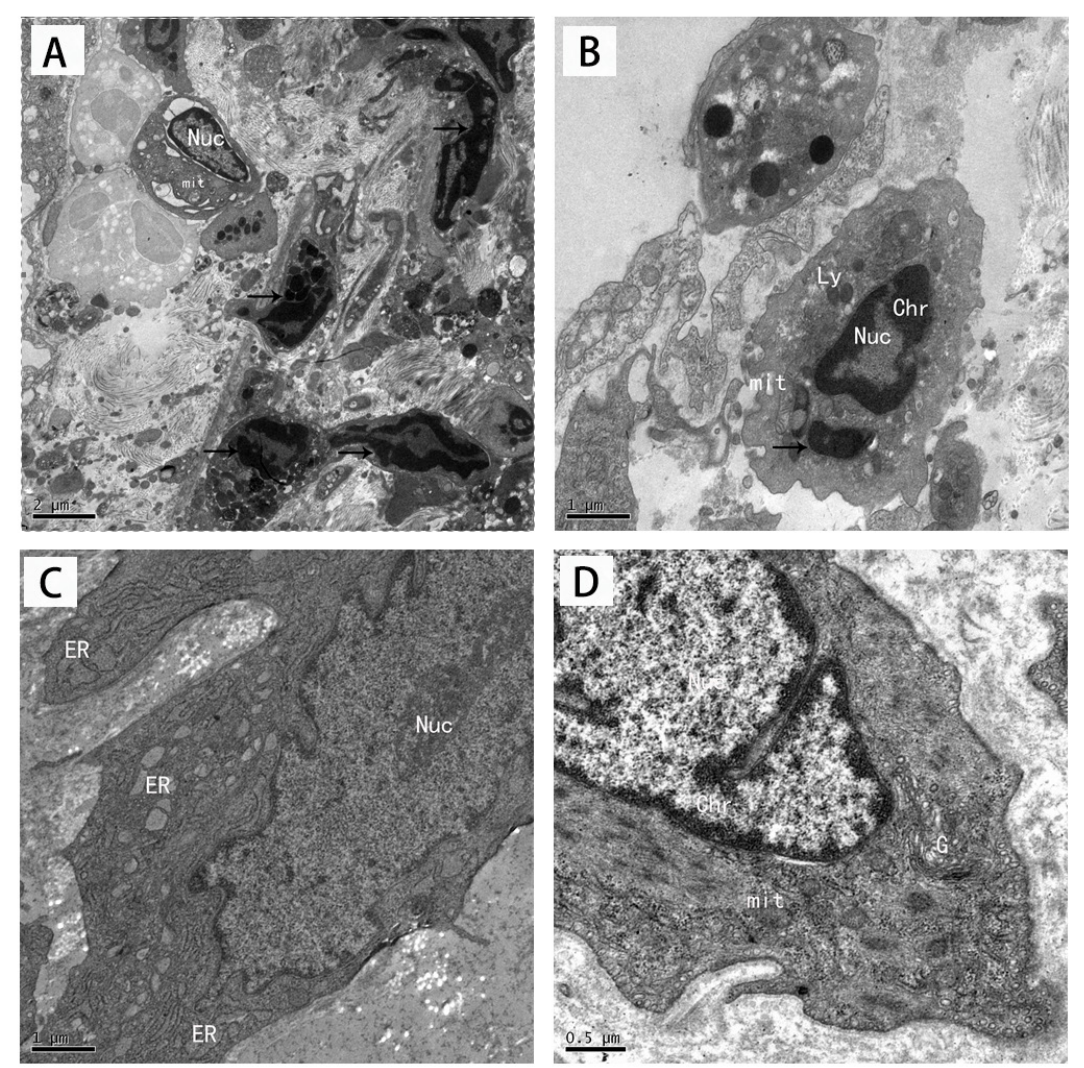

Figure 5. Transmission electron microscopy observation of the vein grafts. In vein grafts on the 1st (A) and 3rd (B) days following surgery, apoptotic bodies were observed. On the 7th $(\mathbf{C})$ and 14th (D) days after surgery, both Golgi apparatus and rough endoplasmic reticulum were abundant in the smooth muscle cells. Arrows indicate apoptotic bodies. mit $=$ mitochondrion, $\mathrm{ER}=$ rough endoplasmic reticulum, $\mathrm{G}=$ Golgi apparatus, $\mathrm{Chr}=$ Chromatin, Nuc $=$ Nucleus, Ly $=$ Lysosomes. Bars $=2 \mu \mathrm{m}(\mathbf{A}), 1 \mu \mathrm{m}(\mathbf{B}$ and $\mathbf{C})$, and $0.5 \mu \mathrm{m}(\mathbf{D})$.

\section{DISCUSSION}

Previous studies have demonstrated that combining a fibrin glue scaffold with oligonucleotides antisense to PCNA results in a synergistic inhibitory effect on the intimal and medial hyperplasia characteristic of grafted veins (Jiang et al., 2007). This effect is associated with maintenance of blood vessel endothelium integrity, inhibition of PCNA expression, facilitation of smooth muscle cell migration, and formation of a new vascular network (Wan 
et al., 2006a,b). However, the long-term consequences are disputed (Stojanovic et al., 2009); therefore, attention must be given to the mechanism underlying restenosis.

During the early period following transplantation (groups T2 and T3), proliferation and intimal thickness both declined slightly on the first day (group T2). It is possible that an incised wound arising from the surgical procedure resulted in injury to the vein graft. In our experiment, TEM demonstrated that apoptosis occurred rapidly following transplantation. During the early period of cell apoptosis, the euchromatin disappeared, and a lack of diffuse distribution was noted. Areas of chromatin were condensed and separated from each other, appearing as well-demarcated, compact blocks adjacent to the karyotheca. On the first day following transplantation (group T2), chromatin margination, nucleolus loss, and apoptotic bodies were observed, demonstrating that apoptosis had occurred.

During the resection of a 4-cm segment of the jugular vein from the surrounding tissue using a non-touch technique, the incised surgical wounds may have caused injury to the vascular endothelial cells and VSMCs, as well as the release of several inflammatory mediators such as TXA2 and adenosine diphosphate. Moreover, after being made part of the arterial system, the vein graft may have suddenly been subjected to high pressures, accompanied by hemodynamic changes and increased wall tension. Excessive expansion of the vein graft might have injured the vascular wall (Myat et al., 2012; Ege et al., 2013). In addition, the shear stress acting on the vein may have increased. These physical and chemical factors could have resulted in apoptosis and stimulated VSMCs in the vessel walls to migrate to the intima (Farris et al., 2011). This may explain the early apoptosis and decline in tunica medial-adventitial thickness observed in the present work following transplantation.

At days 3 and 7 after surgery (groups T3 and T4), proliferation and intimal and tunica medial-adventitial thickness increased, whereas apoptosis decreased. Farris et al. (2011) observed that plasminogen activators, particularly urinary plasminogen activator (uPA), and their receptors play significant roles in vein graft restenosis (Castro and Tanus-Santos, 2013). uPA also facilitates the expression of matrix metalloproteinases (MMPs). Chung et al. (2005) established that the secretion and activity of MMP-2 and MMP-9 in vein grafts is remarkably elevated following transplantation. MMPs and uPA exert synergistic effects, jointly encouraging VSMC migration and proliferation, and causing intimal hyperplasia within the vein graft. The proliferation of medial VSMCs in these grafts may result in the blood shear stress observed under arterial conditions, and decrease the level of apoptosis. Large quantities of synthetic VSMCs were observed by TEM (characterized by increased rough endoplasmic reticulum and Golgi apparatus, and fewer contractile filaments).

Between days 7 and 90 following transplantation (groups T4-T7), the proliferation level declined, while that of apoptosis increased slightly. Moreover, intimal and medialadventitial thicknesses increased substantially. Previous studies have demonstrated that the key steps in restenosis are the proliferation and migration of VSMCs, and the deposition of large amounts of extracellular matrix (ECM). However, VSMCs account for $20 \%$ of intimal hyperplasia, with $60-80 \%$ being made up by ECM (Owens et al., 2015). In this experiment, TEM revealed that ECM expansion was the most striking feature; cell proliferation decreased, and large quantities of collagen fibers were visible. Owens et al. (2015) determined that following coronary artery bypass grafting, thickening of the vein graft wall is caused primarily by collagen production.

Han et al. (1995) studied intimal hyperplasia in rat atherosclerotic vein grafts. They found that the existence of the late apoptosis associated with macrophage, and the inflammatory

Genetics and Molecular Research 15 (4): gmr.15048701 
mediators secreted by macrophage can induce apoptosis. Kim et al. (2012) reported that elevated expression of the gene $B M P-2$ inhibits VSMC proliferation and stimulates their apoptosis, preventing excessive intimal hyperplasia following autologous vein grafting (Fan et al., 2006).

It is widely believed that during the later stages of vein grafting, the promotion of apoptosis may inhibit restenosis of the grafted tissue (Fan et al., 2006; Kim et al., 2012; Liu et al., 2012; Yang et al., 2013). However, in the present study, we found that in a rabbit model of vein graft restenosis, apoptosis was maintained at a low level from the 7th day following transplantation, although the underlying reasons for this are unknown.

Little attention has been paid to the progression of restenosis in vein grafts. In addition, the majority of studies regarding apoptosis have been confined to arterial systems, with only limited consideration of grafted veins. The present experiment is the first of its kind in providing exact time points for the study of the mechanisms of action of both PCNA and apoptosis during grafted vein restenosis, and established a foundation for identifying genetic loci involved in vascular control and remodeling.

\section{Conflicts of interest}

The authors declare no conflict of interest.

\section{ACKNOWLEDGMENTS}

We would like to thank the Department of Pathology of the First Affiliated Hospital of Nanchang University for support during immunohistochemical sectioning. Research supported by the National Natural Science Foundation of China (\#81260055) and the Project of Vein Graft Remodeling of PCNA Protein and Ligand Proteomics.

\section{REFERENCES}

Castro MM and Tanus-Santos JE (2013). Inhibition of matrix metalloproteinases (MMPs) as a potential strategy to ameliorate hypertension-induced cardiovascular alterations. Curr. Drug Targets 14: 335-343.

Chung AW, Rauniyar P, Luo H, Hsiang YN, et al. (2005). Pressure distention compared with pharmacologic relaxation in vein grafting upregulates matrix metalloproteinase-2 and -9. J. Vasc. Surg. 42: 747-756. http://dx.doi.org/10.1016/j. jvs.2005.05.037

Ege MR, Guray U, Guray Y, Ac1kgoz S, et al. (2013). Platelet distribution width and saphenous vein disease in patients after CABG. Association with graft occlusion. Herz 38: 197-201. http://dx.doi.org/10.1007/s00059-012-3668-z

Fan M, Gu TX, Jiang CL and Li Z (2006). Local application of rapamycin inhibiting restenosis in experimental vein graft. Chin. J. Arteriosclerosis 14: 956-958.

Farris SD, Hu JH, Krishnan R, Emery I, et al. (2011). Mechanisms of urokinase plasminogen activator (uPA)-mediated atherosclerosis: role of the uPA receptor and S100A8/A9 proteins. J. Biol. Chem. 286: 22665-22677. http://dx.doi. org/10.1074/jbc.M110.202135

Han DK, Haudenschild CC, Hong MK, Tinkle BT, et al. (1995). Evidence of apoptosis in human atherogenesis and in a rat vascular injury model. Am. J. Pathol. 147: 267-277.

Jeremy JY, Shukla N, Angelini GD and Wan S (2011). Endothelin-1 (ET-1) and vein graft failure and the therapeutic potential of ET-1 receptor antagonists. Pharmacol. Res. 63: 483-489. http://dx.doi.org/10.1016/.j.phrs.2010.10.018

Jiang Z, Yu P, Tao M, Fernandez C, et al. (2007). TGF-beta- and CTGF-mediated fibroblast recruitment influences early outward vein graft remodeling. Am. J. Physiol. Heart Circ. Physiol. 293: H482-H488. http://dx.doi.org/10.1152/ ajpheart.01372.2006

Kim D, Lee D, Jang YL, Chae SY, et al. (2012). Facial amphipathic deoxycholic acid-modified polyethyleneimine for

Genetics and Molecular Research 15 (4): gmr.15048701 
efficient MMP-2 siRNA delivery in vascular smooth muscle cells. Eur. J. Pharm. Biopharm. 81: 14-23. http://dx.doi. org/10.1016/j.ejpb.2012.01.013

Liu CW, Zhang XS and Wang S (2012). Transfection of early growth response gene-1 DNA enzyme mediated by nanoparticles inhibits vein graft intimal hyperplasia. Chin. J. Tissue Eng. Res. 16: 1354-1358.

Myat A, Asrress KN, McConkey H and Redwood SR (2012). Physiological assessment of coronary haemodynamics: fractional flow reserve and beyond. EuroIntervention 7: 1236-1237. http://dx.doi.org/10.4244/EIJV7I10A196

Owens CD, Gasper WJ, Rahman AS and Conte MS (2015). Vein graft failure. J. Vasc. Surg. 61: 203-216. http://dx.doi. org/10.1016/j.jvs.2013.08.019

Stojanovic T, El-Sayed Ahmad A, Didilis V, Ali O, et al. (2009). Extravascular perivenous fibrin support leads to aneurysmal degeneration and intimal hyperplasia in arterialized vein grafts in the rat. Langenbecks Arch. Surg. 394: 357-362. http://dx.doi.org/10.1007/s00423-008-0341-3

Wan L, Li D and Wu Q (2006a). Perivenous application of fibrin glue as external support enhanced adventitial adenovirus transfection in rabbit model. J. Surg. Res. 135: 312-316. http://dx.doi.org/10.1016/j.jss.2006.02.056

Wan L, Li DY, Yang B and Wu QY (2006b). Perivenous application of fibrin glue prevents the early injury of jugular vein graft to arterial circulation in rabbits. Chin. Med. J. (Engl.) 119: 300-304.

Yang J, Zeng Y, Zhang C, Chen YX, et al. (2013). The prevention of restenosis in vivo with a VEGF gene and paclitaxel co-eluting stent. Biomaterials 34: 1635-1643. http://dx.doi.org/10.1016/j.biomaterials.2012.11.006

Genetics and Molecular Research 15 (4): gmr.15048701 